\title{
KINEMATICS AT THE MAIN MECHANISM OF A RAILBOUND FORGING MANIPULATOR
}

Florian Ion Tiberiu Petrescu Bucharest Polytechnic University, Romania E-mail: petrescuflorian@yahoo.com

Relly Victoria Virgil Petrescu Bucharest Polytechnic University, Romania

E-mail: petrescuvictoria@yahoo.com

Submission: $26 / 05 / 2014$

Revision: $14 / 08 / 2014$

Accept: 26/03/2015

\section{ABSTRACT}

Heavy payload forging manipulators are mainly characterized by large load output and large capacitive load input. The relationship between outputs and inputs will greatly influence the control and the reliability. Forging manipulators have become more prevalent in the industry today. They are used to manipulate objects to be forged. The most common forging manipulators are moving on a railway to have a greater precision and stability. They have been called the rail bound forging manipulators. In this paper we analyse general kinematics of the main mechanism from such manipulator. Kinematic scheme shows a typical forging manipulator, with the basic motions in operation process: walking, motion of the tong and buffering. The lifting mechanism consists of several parts including linkages, hydraulic drives and motion pairs. The principle of type design from the viewpoints of the relationship between output characteristics and actuator inputs is discussed. An idea of establishing the incidence relationship between output characteristics and actuator inputs is proposed. These novel forging manipulators which satisfy certain functional requirements provide an effective help for the design of forging manipulators. 
INDEPENDENT JOURNAL OF MANAGEMENT \& PRODUCTION (IJM\&P)

http://www.ijmp.jor.br

v. 6 , n. 3, July - September 2015

ISSN: 2236-269X

DOI: 10.14807/ijmp.v6i3.235

Keywords: Mechatronics, Robotics, Heavy payload forging manipulators, Rail bound forging manipulator, Kinematics

\section{INTRODUCTION}

Heavy payload forging manipulators are mainly characterized by large load output and large capacitive load input. The relationship between outputs and inputs, which will greatly influence the control and the reliability, is the key issue in type design for heavy payload forging manipulators. Forging manipulators have become more prevalent in the industry today. They are used to manipulate objects to be forged [1-3].

The most common forging manipulators are moving on a railway to have a greater precision and stability. They have been called the railbound forging manipulators. In this paper we analyse the general kinematics of the main mechanism from such manipulator [1-5].

Kinematic scheme shows a typical forging manipulator, with the basic motions in operation process: walking, motion of the tong and buffering. The lifting mechanism consists of several parts including linkages, hydraulic drives and motion pairs. Hydraulic drives are with the lifting hydraulic cylinder, the buffer hydraulic cylinder and the leaning hydraulic cylinder, which are individually denoted by c1, c2 and c3. In lifting process, the cylinder $\mathrm{c} 1$ controls the vertical movement of work piece through inputting lifting signal. At the same time, the cylinders c2 and c3 are perfectly closed. While c1 and c3 are closed cylinders, cylinder c2 performs horizontal movement. While, the cylinders $c 1$ and $c 2$ are closed the cylinder c3 realizes leaning movement by inputting leaning signal in leaning condition.

In direct kinematics one knows I1, I2 and must be determined: intermediary I3, $\mathrm{FI} 1, \mathrm{FI}$, FI6, FI8, FI10 and finally $\mathrm{xM}, \mathrm{yM}$. In inverse kinematics one knows xM, yM (imposed) and must be determined FI1, FI3, FI6, FI8, FI10, I1, I2, I3 so that the FI angle keeps its constant value (FI=PI-TETA) to maintain permanently the segment GM horizontally [6-11].

In this work we are solving positions (in inverse kinematics) with systems V, VI. When we know all these parameters (angles and lengths) one may determine all kinematics parameters. The concept of modelling method based on the outputs tasks is defined and investigated. The principle of type design from the viewpoints of the relationship between output characteristics and actuator inputs is discussed. An idea of establishing the incidence relationship between output characteristics and actuator 


\section{INDEPENDENT JOURNAL OF MANAGEMENT \& PRODUCTION (IJM\&P)}

http://www.ijmp.jor.br

v. 6, n. 3, July - September 2015

ISSN: 2236-269X

DOI: 10.14807/ijmp.v6i3.235

inputs is proposed. These novel forging manipulators which satisfy certain functional requirements provide an effective help for the design of forging manipulators [1-5].

In the next 14 photos one can see some forging manipulators (independent or on rail) at work [1-5, 11-12].

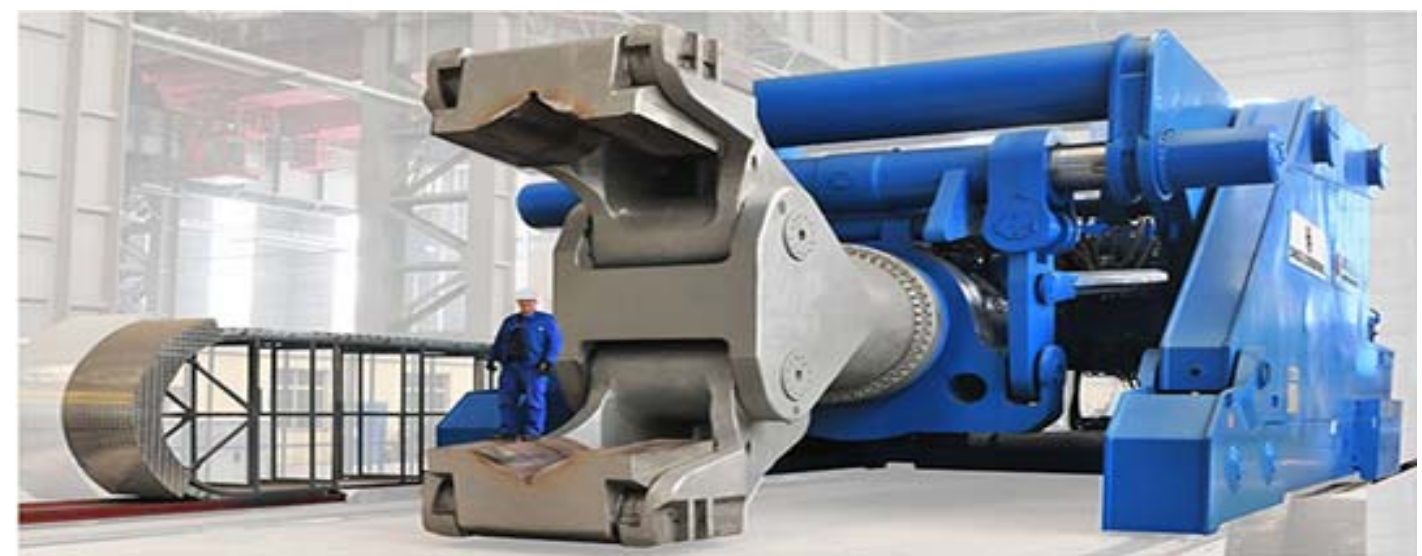

Figure 1: Forging Manipulator (Rail Mobile) Source: Dango \& Dienenthal

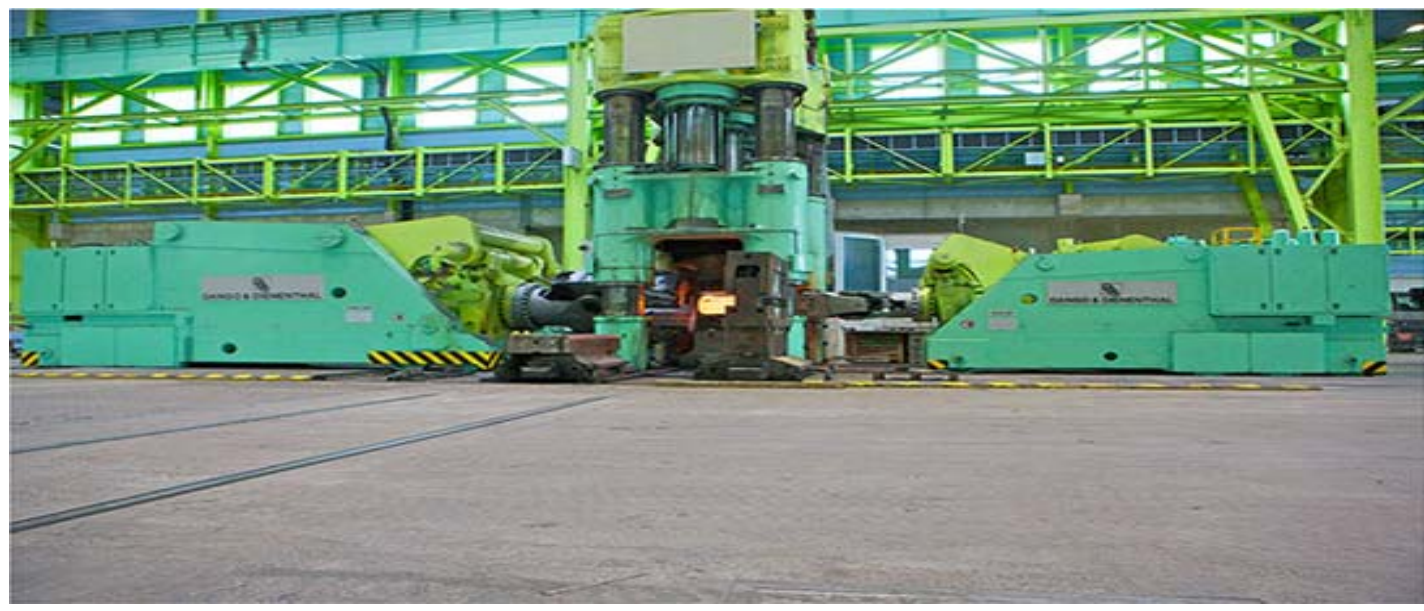

Figure 2: Forging Manipulator (Rail Mobile) Source: Dango \& Dienenthal

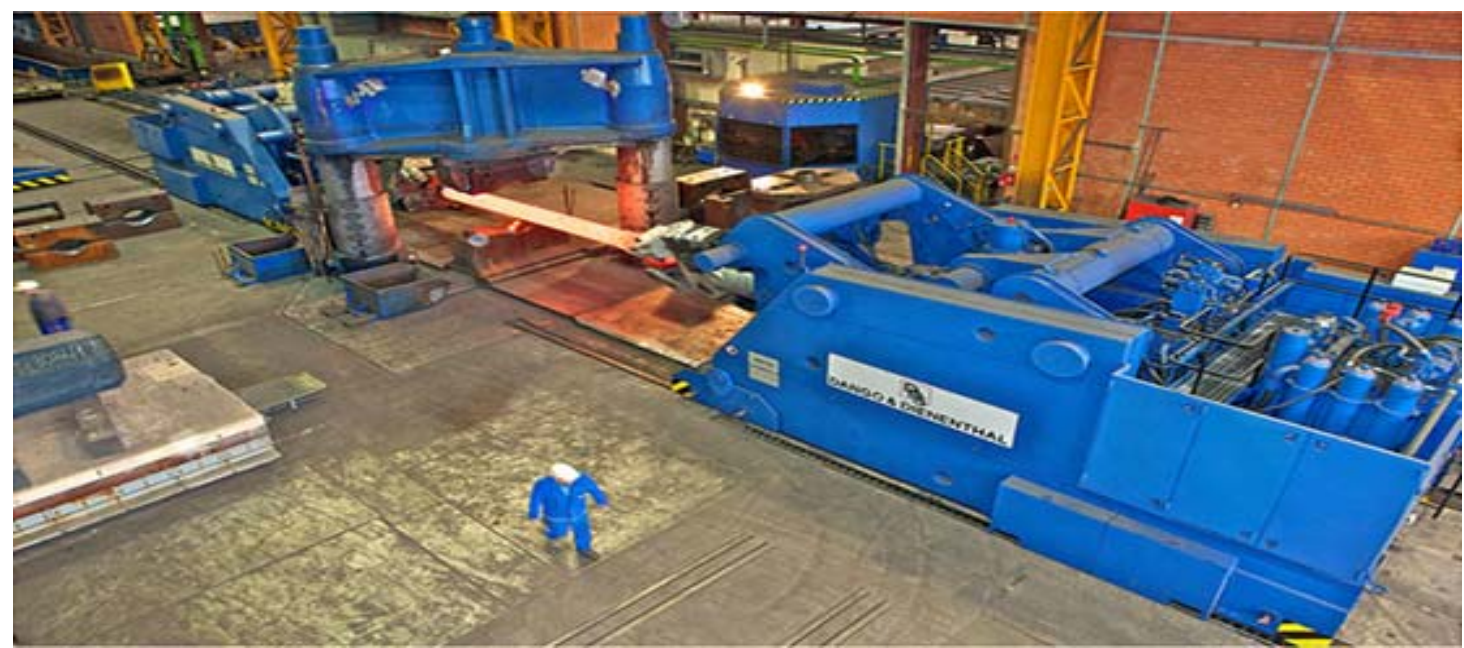

Figure 3: Forging Manipulator (Rail Mobile)

Source: Dango \& Dienenthal 
INDEPENDENT JOURNAL OF MANAGEMENT \& PRODUCTION (IJM\&P)

http://www.ijmp.jor.br

v. 6, n. 3, July - September 2015

ISSN: 2236-269X

DOI: 10.14807/ijmp.v6i3.235

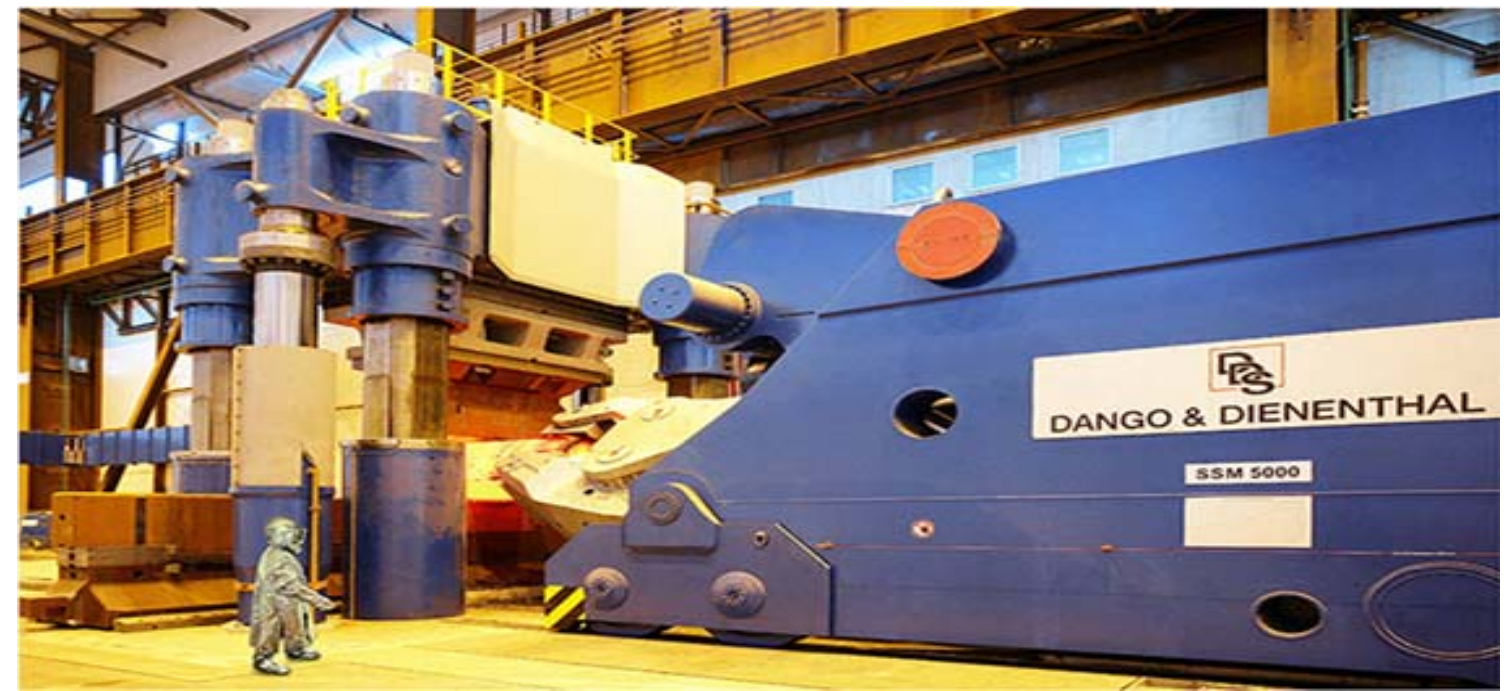

Figure 4: Forging Manipulator (Rail Mobile) Source: Dango \& Dienenthal

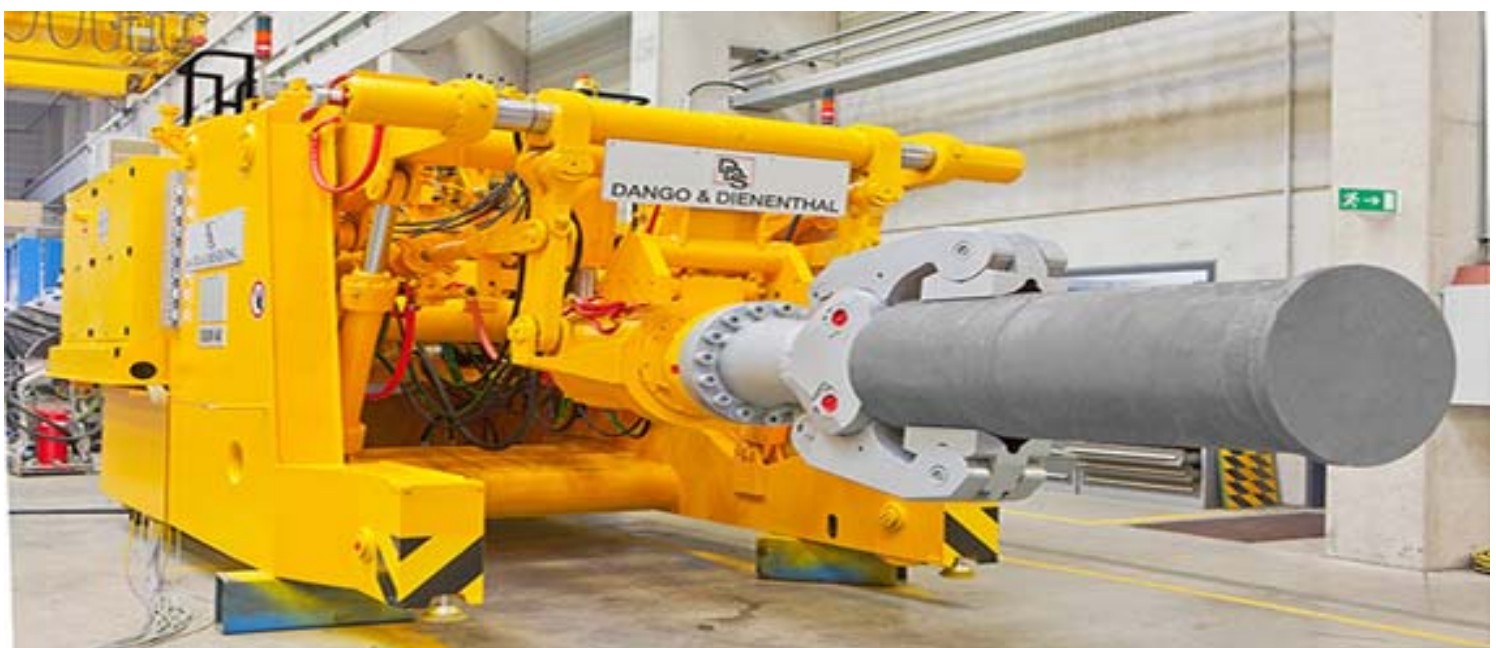

Figure 5: Forging Manipulator (Mobile)

Source: Dango \& Dienenthal

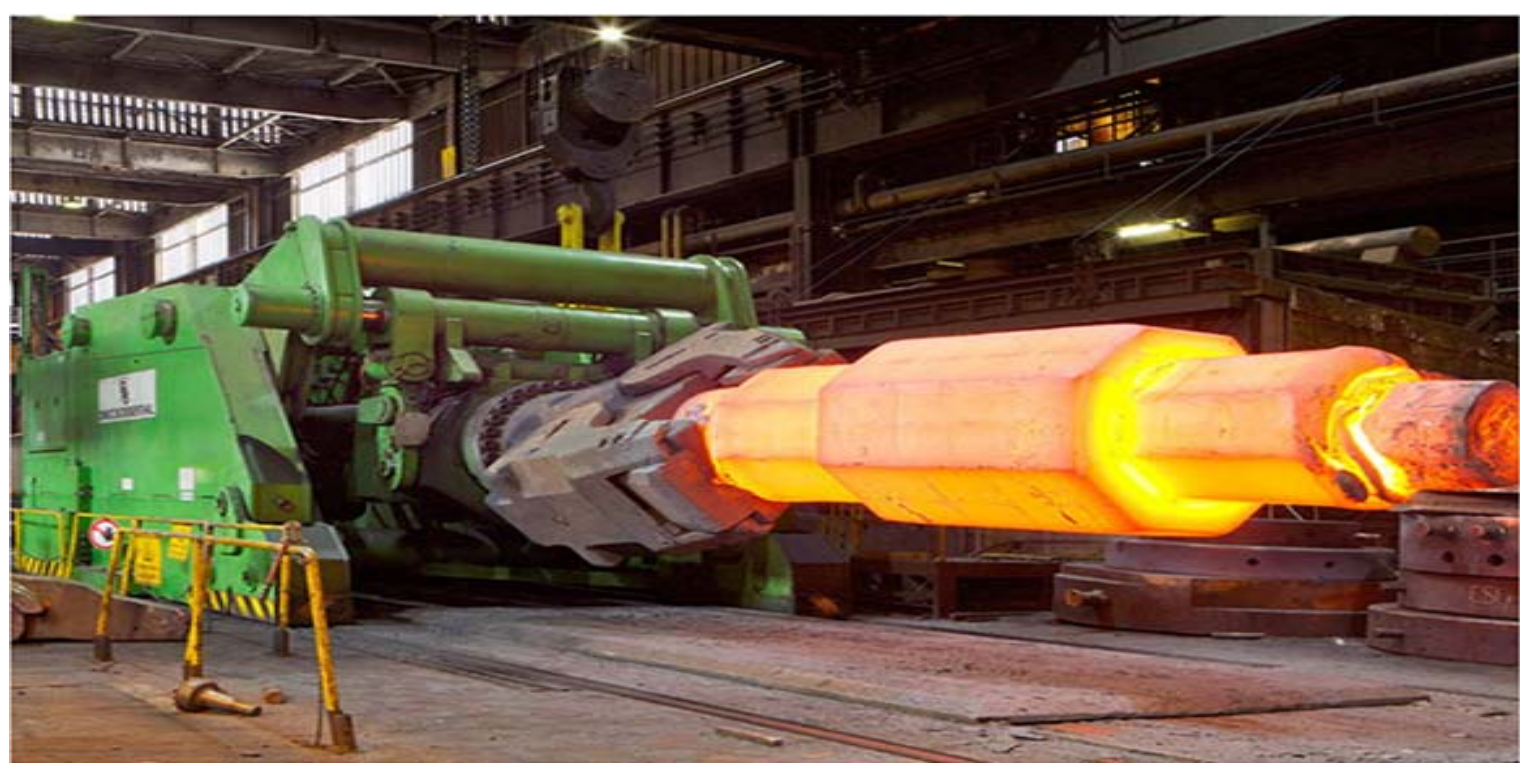

Figure 6: Forging Manipulator (Rail Mobile)

Source: Dango \& Dienenthal 
ISSN: 2236-269X

DOI: $10.14807 /$ ijmp.v6i3.235

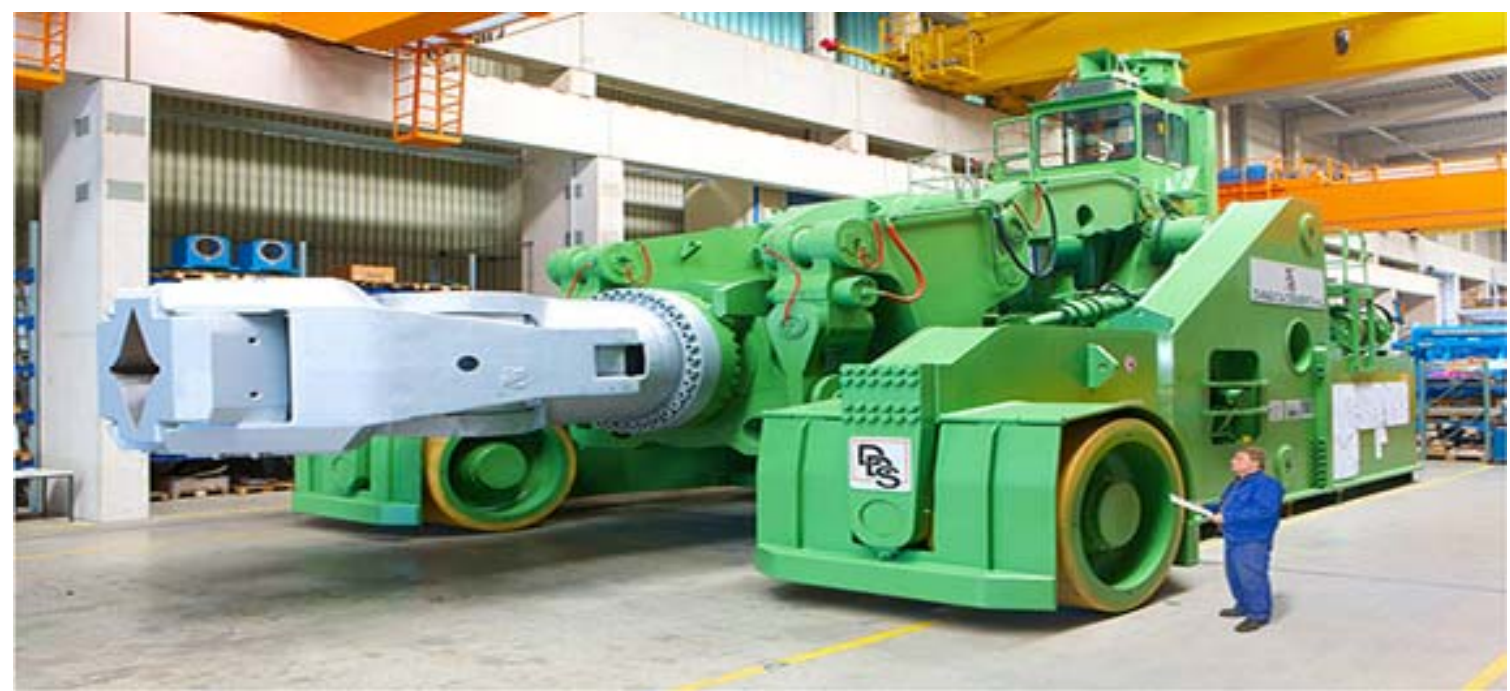

Figure 7: Forging Manipulator (Independent Mobile) Source: Dango \& Dienenthal

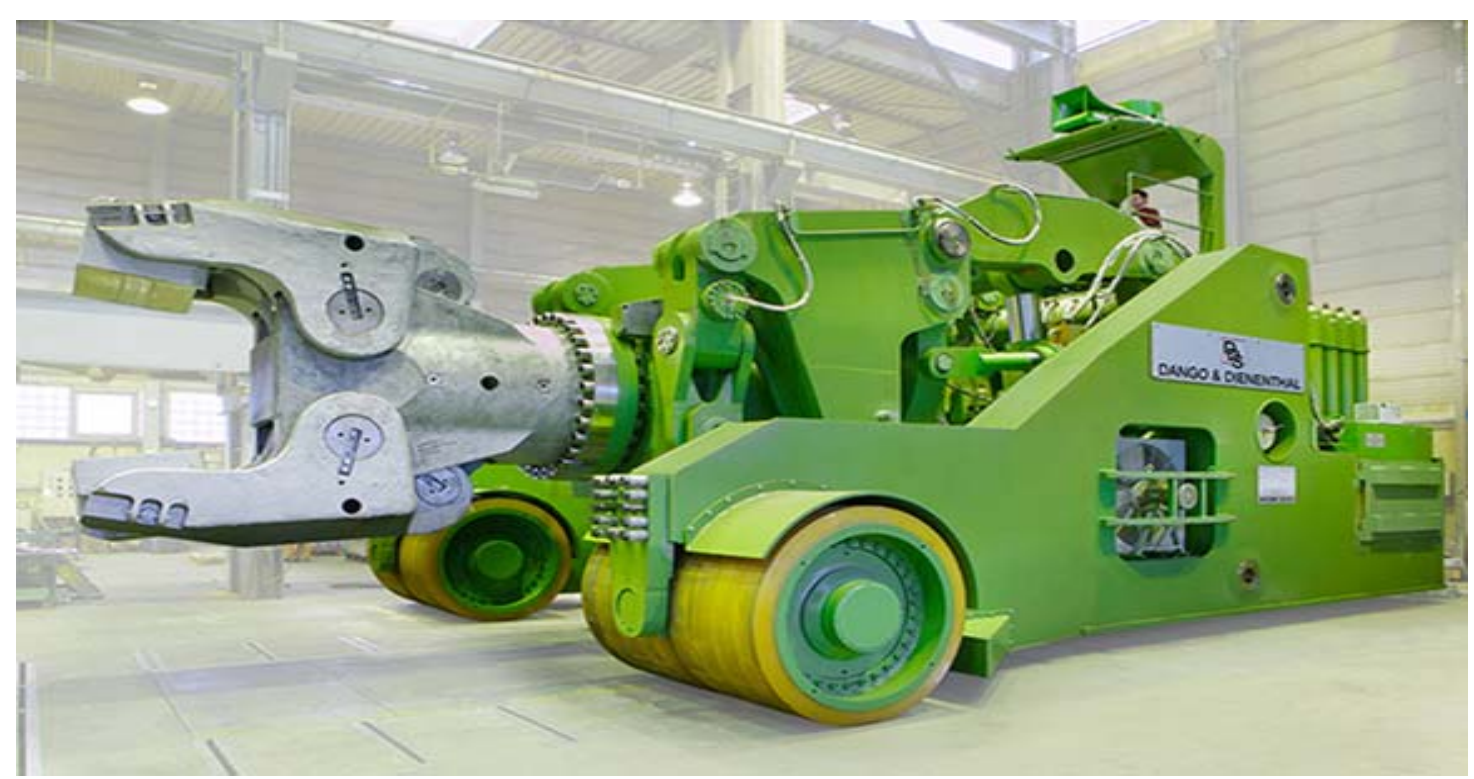

Figure 8: Forging Manipulator (Independent Mobile) Source: Dango \& Dienenthal

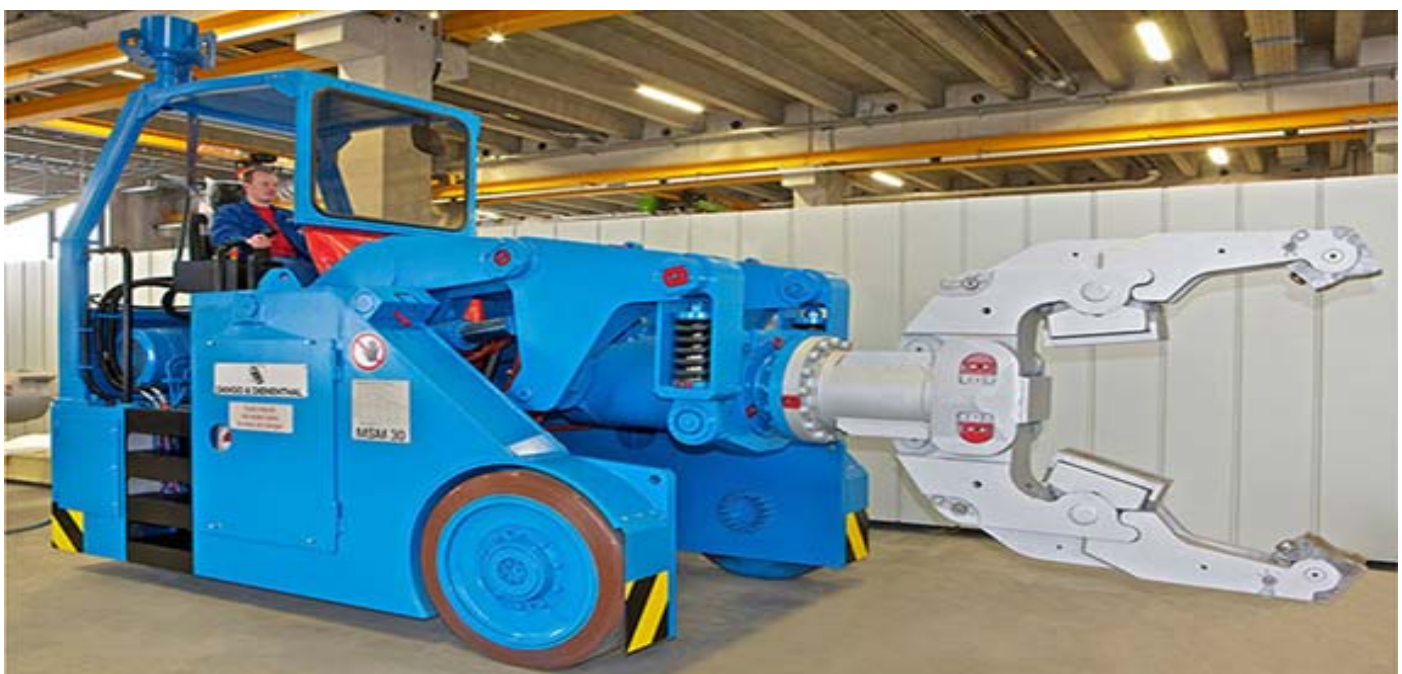

Figure 9: Forging Manipulator (Independent Mobile)

Source: Dango \& Dienenthal 
INDEPENDENT JOURNAL OF MANAGEMENT \& PRODUCTION (IJM\&P)

http://www.ijmp.jor.br

v. 6, n. 3, July - September 2015

ISSN: 2236-269X

DOI: $10.14807 /$ ijmp.v6i3.235

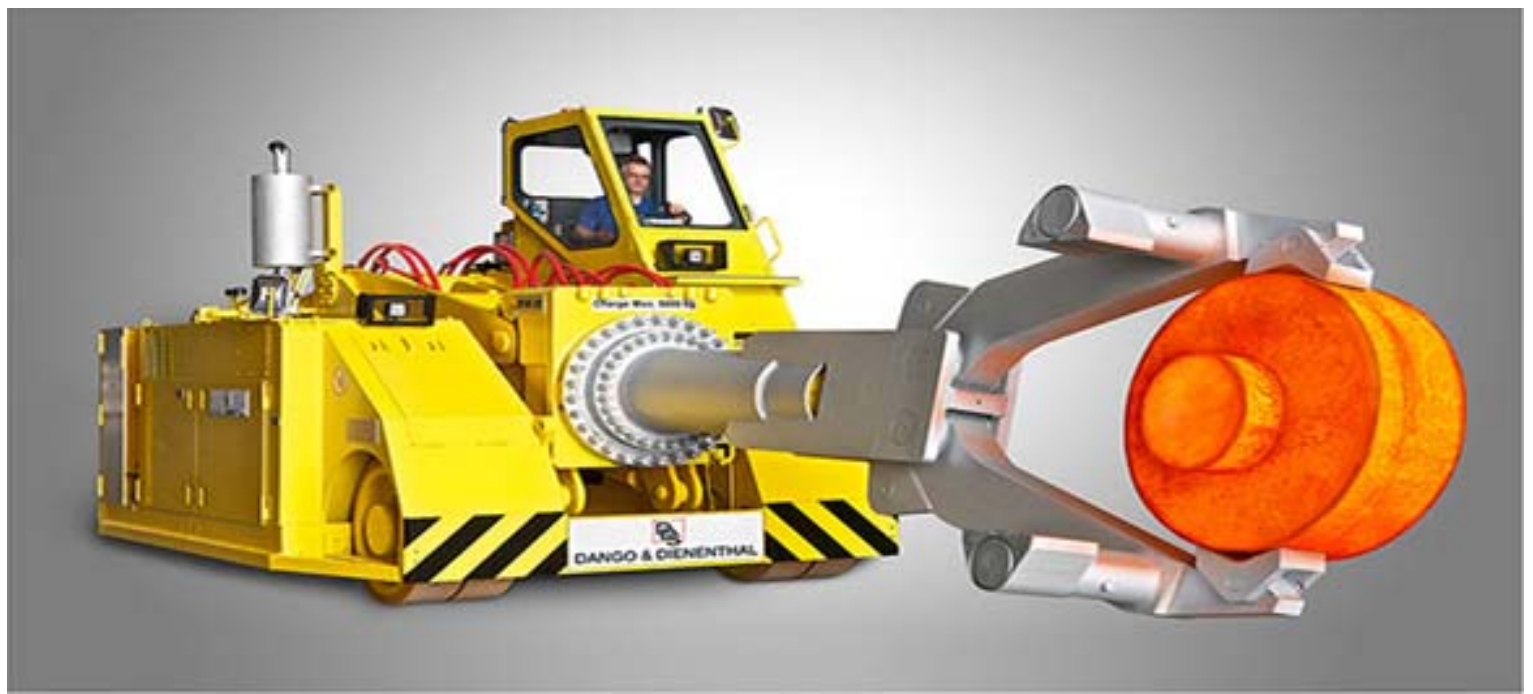

Figure 10: Forging Manipulator (Independent Mobile) Source: Dango \& Dienenthal

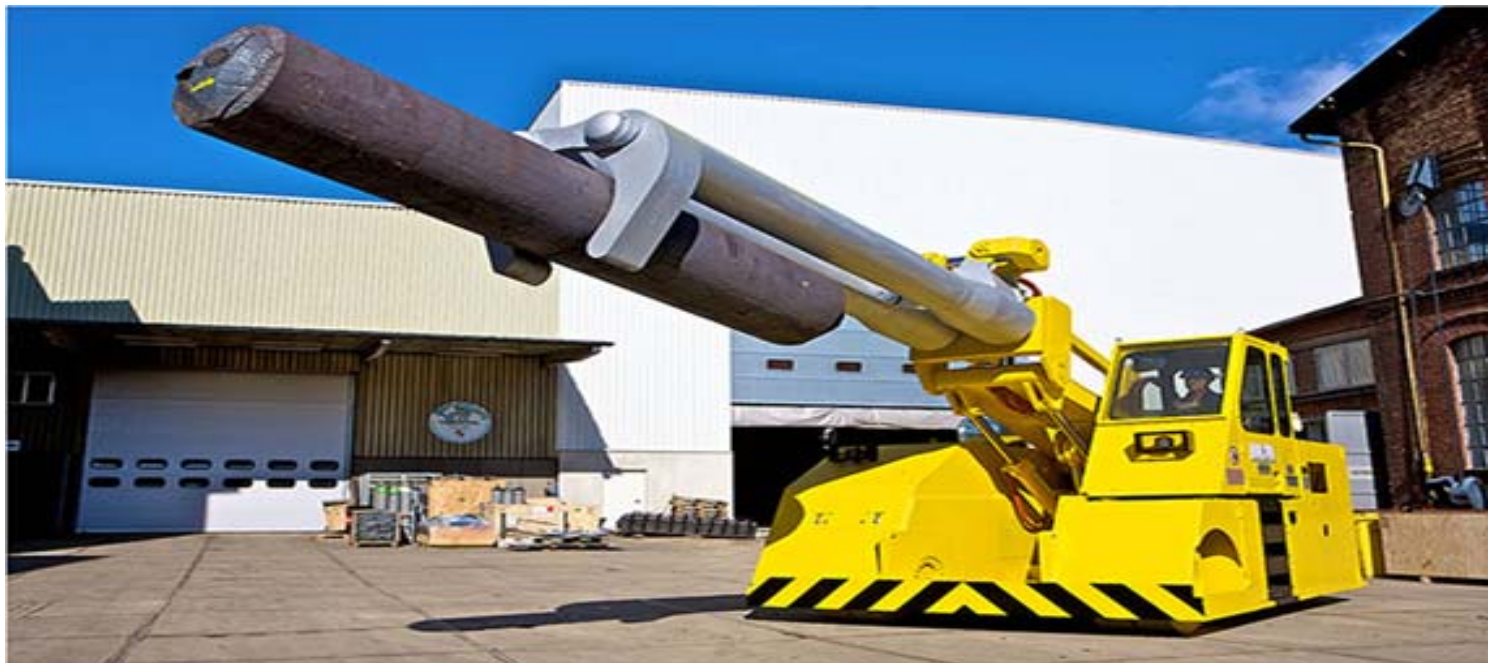

Figure 11: Forging Manipulator (Independent Mobile) Source: Dango \& Dienenthal

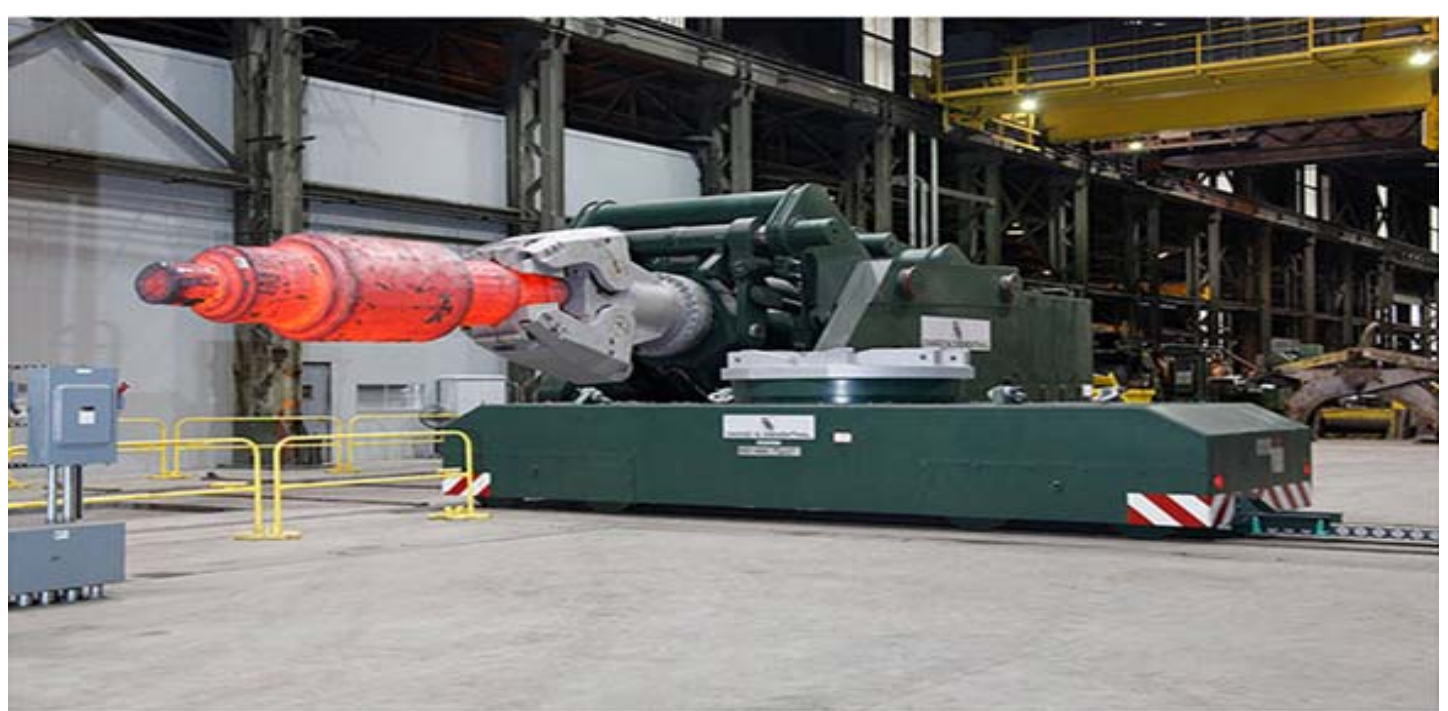

Figure 12: Forging Manipulator (Rail Mobile)

Source: Dango \& Dienenthal 


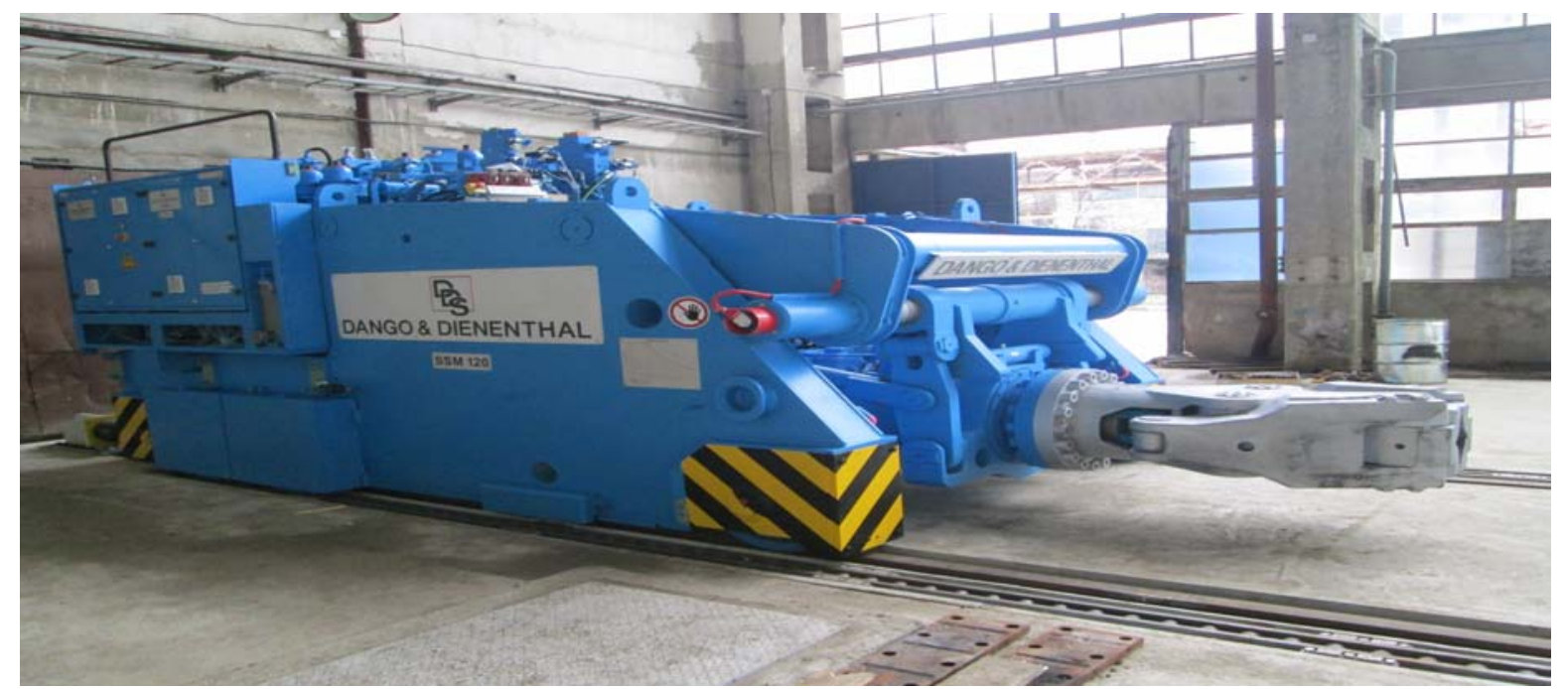

Figure 13: Forging Manipulator (Rail Mobile) Source: Dango \& Dienenthal

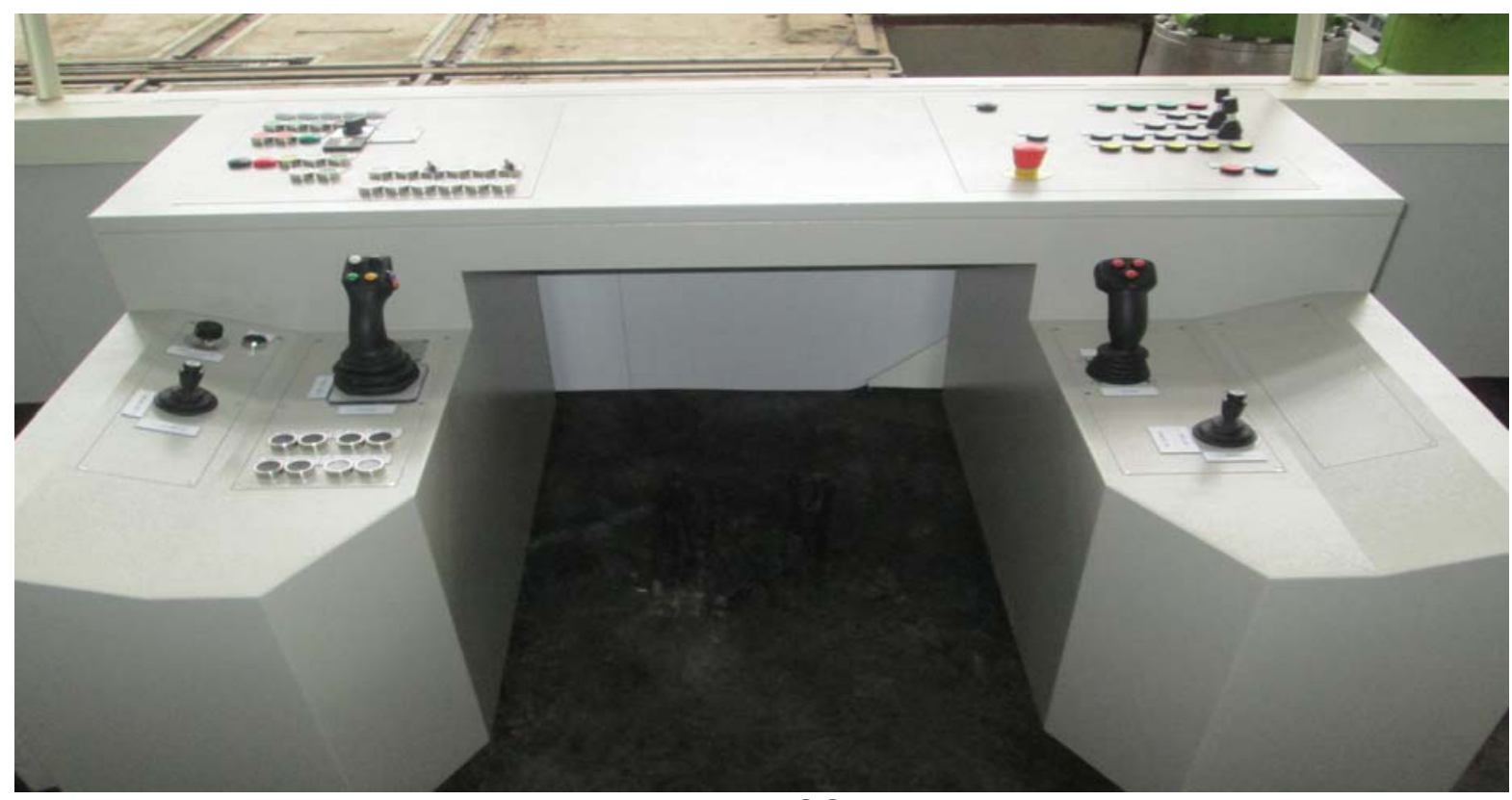

Figure 14: Workstation SSM120 equipment Source: Dango \& Dienenthal

\subsection{Nomenclature}

$\mathrm{c}_{1}$ - lifting hydraulic cylinder; $\mathrm{c}_{2}$ - the buffer hydraulic cylinder;

$\mathrm{C}_{3}$ - leaning hydraulic cylinder; $\mathrm{I}_{1}, \mathrm{I}_{2}, \mathrm{I}_{3}$ - variable lengths;

A-L - linkages; A, B, K, F - fixed linkages; $\varphi_{1}, \varphi_{3}, \varphi_{6}, \varphi_{8}, \varphi_{10}$ - variable angles; a-g - constant lengths; $\mathrm{x}_{\mathrm{B}}, \mathrm{y}_{\mathrm{B}}, \mathrm{x}_{\mathrm{A}}, \mathrm{y}_{\mathrm{A}}, \mathrm{x}_{\mathrm{K}}, \mathrm{y}_{\mathrm{K}}, \mathrm{x}_{\mathrm{F}}, \mathrm{y}_{\mathrm{F}}$ - constant coordinates; $\beta, \theta, \varphi_{4}-$ constant angles; $\varphi$ - an angle which must be maintained constant $(\varphi=\pi-\theta)$ to keep permanently the segment GM horizontally (as shown in Figure 15). 
INDEPENDENT JOURNAL OF MANAGEMENT \& PRODUCTION (IJM\&P)

http://www.ijmp.jor.br

v. 6, n. 3, July - September 2015

ISSN: 2236-269X

DOI: 10.14807/ijmp.v6i3.235

\section{THE STRUCTURE, GEOMETRY AND KINEMATICS OF A RAILBOUND FORGING MANIPULATOR}

In fig. 15 one can see the kinematics schema of the main mechanism from a railbound forging manipulator [11].

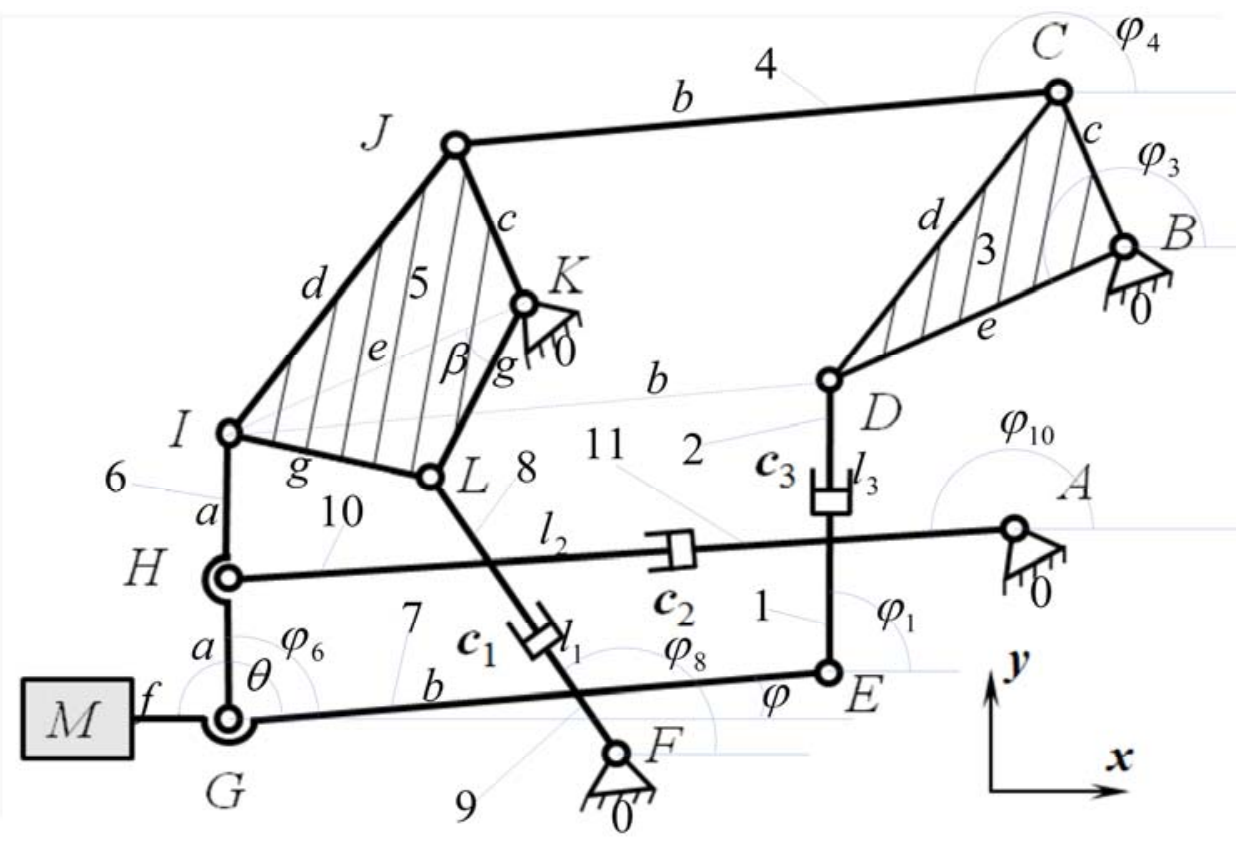

Figure 15: Cinematic schema of a forging manipulator main mechanism

Permanently one knows the constant lengths (a-g) (a to b)and the coordinates $\left(\mathrm{x}_{\mathrm{B}}, \mathrm{y}_{\mathrm{B}}, \mathrm{x}_{\mathrm{A}}, \mathrm{y}_{\mathrm{A}}, \mathrm{x}_{\mathrm{K}}, \mathrm{y}_{\mathrm{K}}, \mathrm{x}_{\mathrm{F}}, \mathrm{y}_{\mathrm{F}}\right.$ ), (not identify the coordinated) and the $\varphi$ angle who must to be maintained constant [1-5, 9-12]. (constant angle of 1 to 3 or 1 and 3)

In direct kinematics one knows $I_{1}, I_{2}$ and must be determined: intermediary (with systems I, II, III) I $I_{3}, \varphi_{1}, \varphi_{3}, \varphi_{6}, \varphi_{8}, \varphi_{10}$ and finaly (with system IV) $x_{M}, y_{M}[1-5,9-$ 12].

In inverse kinematics one knows $x_{M}, y_{M}$ and must be determined $\varphi_{1}, \varphi_{3}, \varphi_{6}, \varphi_{8}$, $\varphi_{10}, I_{1}, I_{2}, I_{3}$ with systems I, II, III, IV.

It takes four independent vector contours (KLFK, KIGEDB, AHIK, AHGM) and one can write the below systems (I, II, III, IV) [1-5, 9-12].

$$
\left\{\begin{array}{l}
\left(x_{K}-x_{F}\right)+g \cdot \cos \left(\varphi_{3}+\beta\right)=l_{1} \cdot \cos \varphi_{8} \\
\left(y_{K}-y_{F}\right)+g \cdot \sin \left(\varphi_{3}+\beta\right)=l_{1} \cdot \sin \varphi_{8}
\end{array}\right.
$$




$$
\begin{aligned}
& \left\{\begin{array}{l}
x_{K}+b \cdot \cos \varphi+l_{3} \cdot \cos \varphi_{1}=2 a \cdot \cos \varphi_{6} \\
y_{K}+b \cdot \sin \varphi+l_{3} \cdot \sin \varphi_{1}=2 a \cdot \sin \varphi_{6}
\end{array}\right. \\
& \left\{\begin{array}{l}
\left(x_{A}-x_{K}\right)+l_{2} \cdot \cos \varphi_{10}+a \cdot \cos \varphi_{6}=e \cdot \cos \varphi_{3} \\
\left(y_{A}-y_{K}\right)+l_{2} \cdot \sin \varphi_{10}+a \cdot \sin \varphi_{6}=e \cdot \sin \varphi_{3}
\end{array}\right. \\
& \left\{\begin{array}{l}
\left(x_{A}-x_{M}\right)+l_{3} \cdot \cos \varphi_{10}+f \cdot \cos (\varphi+\theta)=a \cdot \cos \varphi_{6} \\
\left(y_{A}-y_{M}\right)+l_{3} \cdot \sin \varphi_{10}+f \cdot \sin (\varphi+\theta)=a \cdot \sin \varphi_{6}
\end{array}\right.
\end{aligned}
$$

\subsection{Inverse kinematics relationships computing}

Then can be determined easily the parameters $\varphi_{1}, \varphi_{3}, \varphi_{6}, \varphi_{8}, \varphi_{10}, I_{1}, I_{2}, l_{3}$ solving the four systems I, II, III, IV. Following relationships (systems V, VI) are obtained [1-5, 9-12].

$$
\begin{aligned}
& \int \cos \varphi_{6}=\frac{A_{1} \cdot A_{2} \mp A_{3} \cdot \sqrt{A_{2}^{2}+A_{3}^{2}-A_{1}^{2}}}{A_{2}^{2}+A_{3}^{2}} ; \varphi_{6}=\arccos \left(\cos \varphi_{6}\right) \\
& A_{0}=4 a^{2}+\left(x_{K}+b \cos \varphi\right)^{2}+\left(y_{K}+b \sin \varphi\right)^{2}- \\
& -4 a\left[\left(x_{K}+b \cos \varphi\right) \cos \varphi_{6}+\left(y_{K}+b \sin \varphi\right) \sin \varphi_{6}\right] ; \quad l_{3}=\sqrt{A_{0}} \\
& \left\{\begin{array}{l}
\cos \varphi_{1}=\frac{2 a \cdot \cos \varphi_{6}-x_{K}-b \cdot \cos \varphi}{l_{3}} \\
\sin \varphi_{1}=\frac{2 a \cdot \sin \varphi_{6}-y_{K}-b \cdot \sin \varphi}{l_{3}}
\end{array} ;\left\{\begin{array}{l}
\varphi_{1}=\operatorname{sign}\left(\sin \varphi_{1}\right) . \\
\arccos \left(\cos \varphi_{1}\right)
\end{array}\right.\right. \\
& \cos \varphi_{10}=\frac{a \cdot \cos \varphi_{6}-f \cdot \cos (\varphi+\theta)+x_{M}-x_{A}}{l_{3}} \\
& \sin \varphi_{10}=\frac{a \cdot \sin \varphi_{6}-f \cdot \sin (\varphi+\theta)+y_{M}-y_{A}}{l_{3}} \\
& \varphi_{10}=\operatorname{sign}\left(\sin \varphi_{10}\right) \cdot \arccos \left(\cos \varphi_{10}\right) \\
& l_{2}=-A_{4} \mp \sqrt{A_{4}^{2}+e^{2}}
\end{aligned}
$$




$$
\left\{\begin{array}{l}
\left\{\begin{array}{l}
\cos \varphi_{3}=\frac{x_{A}-x_{K}+l_{2} \cdot \cos \varphi_{10}+a \cdot \cos \varphi_{6}}{e} \\
\sin \varphi_{3}=\frac{y_{A}-y_{K}+l_{2} \cdot \sin \varphi_{10}+a \cdot \sin \varphi_{6}}{e}
\end{array}\right. \\
\Rightarrow \varphi_{3}=\operatorname{sign}\left(\sin \varphi_{3}\right) \cdot \arccos \left(\cos \varphi_{3}\right) \\
l_{1}= \\
=\sqrt{\left[x_{K}-x_{F}+g \cos \left(\varphi_{3}+\beta\right)\right]^{2}+\left[y_{K}-y_{F}+g \sin \left(\varphi_{3}+\beta\right)\right]^{2}} \\
\left\{\begin{array}{l}
\cos \varphi_{8}=\frac{x_{K}-x_{F}+g \cdot \cos \left(\varphi_{3}+\beta\right)}{l_{1}} \\
\sin \varphi_{8}=\frac{y_{K}-y_{F}+g \cdot \sin \left(\varphi_{3}+\beta\right)}{l_{1}} \Rightarrow \\
\Rightarrow \varphi_{8}=\operatorname{sign}\left(\sin \varphi_{8}\right) \cdot \arccos \left(\cos \varphi_{8}\right) \\
A_{1}=3 a^{2}+\left(x_{K}+b \cdot \cos \varphi\right)^{2}+\left(y_{K}+b \cdot \sin \varphi\right)^{2}- \\
-\left[x_{M}-x_{A}-f \cdot \cos (\varphi+\theta)\right]^{2}-\left[y_{M}-y_{A}-f \cdot \sin (\varphi+\theta)\right]^{2} \\
A_{2}=4 a \cdot\left(x_{K}+b \cdot \cos \varphi\right)+2 a \cdot\left[x_{M}-x_{A}-f \cdot \cos (\varphi+\theta)\right] \\
A_{3}=4 a \cdot\left(y_{K}+b \cdot \sin \varphi\right)+2 a \cdot\left[y_{M}-y_{A}-f \cdot \sin (\varphi+\theta)\right] \\
A_{4}=\cos \varphi_{10} \cdot\left(a \cdot \cos \varphi_{6}+x_{A}-x_{K}\right)+\sin \varphi_{10} \cdot \\
\cdot\left(a \cdot \sin \varphi_{6}+y_{A}-y_{K}\right)
\end{array}\right.
\end{array}\right.
$$

\subsection{General kinematics relationships computing}

In the fig. 16 one can see the general kinematics schema of a railbound forging manipulator main mechanism [11].

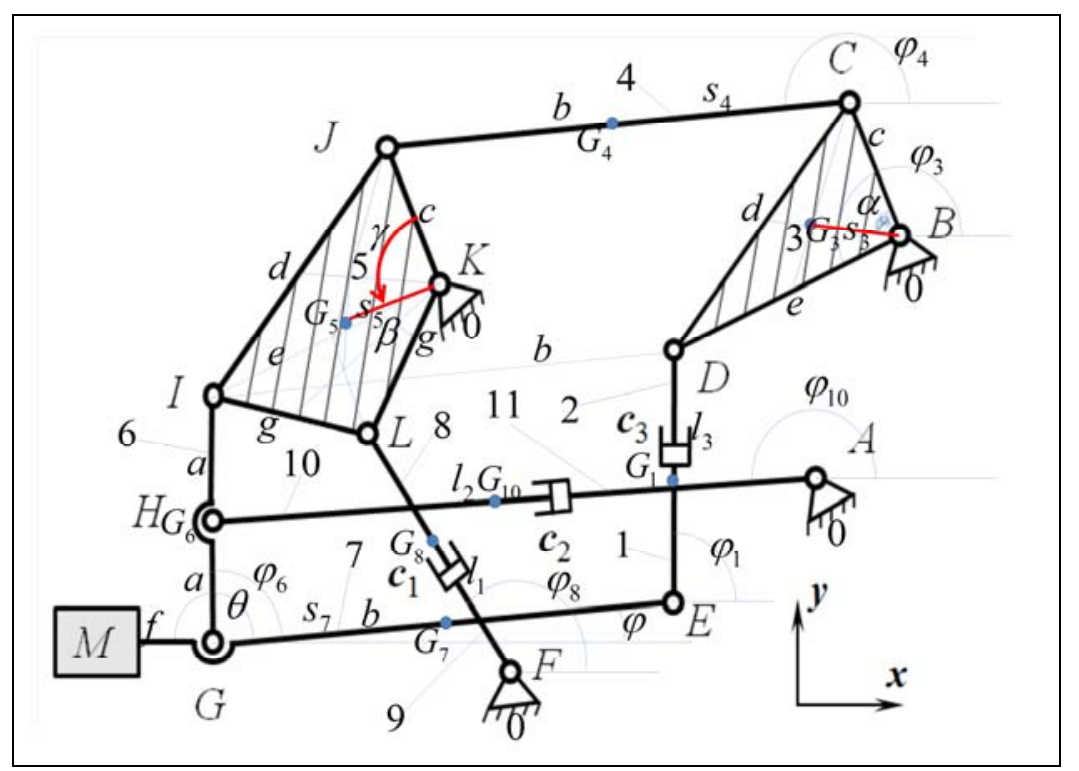

Figure 16: General cinematic schema of a forging manipulator main mechanism 
At the first step, starting from the system I derived by time (in function of time), one calculates the angular velocities $\dot{\varphi}_{3}, \dot{\varphi}_{8}$ in function of the linear velocity of the engine $c 1, i_{1}$ (see the system 1) [11].

$$
\left\{\begin{array}{l}
\left\{\begin{array}{c}
\left|\begin{array}{c}
I a \\
-g \cdot \sin \left(\varphi_{3}+\beta\right) \cdot \dot{\varphi}_{3}=-l_{1} \cdot \sin \varphi_{8} \cdot \dot{\varphi}_{8}+\cos \varphi_{8} \cdot i_{1} \\
g \cdot \cos \left(\varphi_{3}+\beta\right) \cdot \dot{\varphi}_{3}=l_{1} \cdot \cos \varphi_{8} \cdot \dot{\varphi}_{8}+\sin \varphi_{8} \cdot \dot{l}_{1}
\end{array}\right| \begin{array}{c}
I b \\
\sin \varphi_{8}
\end{array} \| \cdot \cos \left(\varphi_{3}+\beta\right) \\
\sin \left(\varphi_{3}+\beta\right)
\end{array} \|\right. \\
I a \Rightarrow \dot{\varphi}_{3} \cdot g \cdot \sin \left(\varphi_{8}-\varphi_{3}-\beta\right)=\dot{l}_{1} \Rightarrow \dot{\varphi}_{3}=\frac{i_{1}}{g \cdot \sin \left(\varphi_{8}-\varphi_{3}-\beta\right)} \\
\varphi_{8}-\varphi_{3}-\beta \neq k \pi \\
I b \Rightarrow \dot{\varphi}_{8} \cdot l_{1} \cdot \sin \left(\varphi_{8}-\varphi_{3}-\beta\right)=\cos \left(\varphi_{8}-\varphi_{3}-\beta\right) \cdot \dot{l}_{1} \Rightarrow \\
\Rightarrow \dot{\varphi}_{8}=\frac{\cos \left(\varphi_{8}-\varphi_{3}-\beta\right) \cdot \dot{l}_{1}}{l_{1} \cdot \sin \left(\varphi_{8}-\varphi_{3}-\beta\right)} \quad \varphi_{8}-\varphi_{3}-\beta \neq k \pi
\end{array}\right.
$$

At the step two, starting from the system II derivated by time, one calculates the angular velocities $\dot{\varphi}_{6}, \dot{\varphi}_{10}$ in function of linear velocities $\dot{l}_{1}, \dot{l}_{2}$ of engines c1, c2 (resulting the system 2). Solving every sytem is simple and direct; multiply at the step a the first equation with a cosine and the second equation with a sine, one add the two relations rezulted and one obtains an equation linear grade 1 with a single unknown. To the pass $b$ one repeat the procedure but the multiply of the two equations is different [1-4, 9-11]. 


$$
\begin{aligned}
& \left\{\left\{\begin{array}{c|c}
\cos \varphi_{10} \dot{l}_{2}-l_{2} \sin \varphi_{10} \dot{\varphi}_{10}-a \sin \varphi_{6} \dot{\varphi}_{6}=-e \sin \varphi_{3} \dot{\varphi}_{3} \\
\sin \varphi_{10} \dot{l}_{2}+l_{2} \cos \varphi_{10} \dot{\varphi}_{10}+a \cos \varphi_{6} \dot{\varphi}_{6}=e \cos \varphi_{3} \dot{\varphi}_{3}
\end{array} \mid \cdot \begin{array}{c}
I I b \\
\sin \varphi_{10}
\end{array}\left\|\cdot \begin{array}{c}
\cos \varphi_{6} \\
\sin \varphi_{6}
\end{array}\right\|\right.\right. \\
& \text { II } \Rightarrow \dot{l}_{2}+a \cdot \sin \left(\varphi_{10}-\varphi_{6}\right) \cdot \dot{\varphi}_{6}=e \cdot \sin \left(\varphi_{6}-\varphi_{3}\right) \cdot \dot{\varphi}_{3} \Rightarrow \\
& \Rightarrow \dot{\varphi}_{6}=\frac{e \cdot \sin \left(\varphi_{6}-\varphi_{3}\right) \cdot \dot{\varphi}_{3}-\dot{l}_{2}}{a \cdot \sin \left(\varphi_{10}-\varphi_{6}\right)} \quad \varphi_{10}-\varphi_{6} \neq k \pi \\
& I I b \Rightarrow \cos \left(\varphi_{10}-\varphi_{6}\right) \cdot \dot{l}_{2}-l_{2} \cdot \sin \left(\varphi_{10}-\varphi_{6}\right) \cdot \dot{\varphi}_{10}=e \cdot \sin \left(\varphi_{6}-\varphi_{3}\right) \cdot \dot{\varphi}_{3} \Rightarrow \\
& \Rightarrow \dot{\varphi}_{10}=\frac{\cos \left(\varphi_{10}-\varphi_{6}\right) \cdot \dot{l}_{2}-e \cdot \sin \left(\varphi_{6}-\varphi_{3}\right) \cdot \dot{\varphi}_{3}}{l_{2} \cdot \sin \left(\varphi_{10}-\varphi_{6}\right)} \quad \varphi_{10}-\varphi_{6} \neq k \pi
\end{aligned}
$$

At the step three, starting from the system III derivated by time, it calculates the angular velocity $\dot{\varphi}_{1}$ in function of linear velocities $i_{1}, i_{2}$ of the engines (actuators) c1, c2 (result the system 3) [6-11].

$$
\left\{\begin{array}{l}
\left\{\begin{array}{l}
I I I a \\
l_{3} \cdot \cos \varphi_{1}-l_{3} \cdot \sin \varphi_{1} \cdot \dot{\varphi}_{1}=-2 a \cdot \sin \varphi_{6} \cdot \dot{\varphi}_{6} \\
\dot{l}_{3} \cdot \sin \varphi_{1}+l_{3} \cdot \cos \varphi_{1} \cdot \dot{\varphi}_{1}=2 a \cdot \cos \varphi_{6} \cdot \dot{\varphi}_{6}
\end{array} \mid \cdot \sin \varphi_{1}\right. \\
\text { IIIa } \Rightarrow l_{3} \cdot \dot{\varphi}_{1}=2 a \cdot \cos \varphi_{1}
\end{array} \mid\right.
$$

At the step four we arrange the system IV and then one derivated it by time and it obtains directly the scalar velocities of the endeffector point M (system 4) [11].

$$
\left\{\begin{array}{l}
\left\{\begin{array}{l}
x_{M}=l_{3} \cdot \cos \varphi_{10}+f \cdot \cos (\varphi+\theta)-a \cdot \cos \varphi_{6} \\
y_{M}=l_{3} \cdot \sin \varphi_{10}+f \cdot \sin (\varphi+\theta)-a \cdot \sin \varphi_{6}
\end{array}\right. \\
\left\{\begin{array}{l}
\dot{x}_{M}=\dot{l}_{3} \cdot \cos \varphi_{10}-l_{3} \cdot \sin \varphi_{10} \cdot \dot{\varphi}_{10}+a \cdot \sin \varphi_{6} \cdot \dot{\varphi}_{6} \\
\dot{y}_{M}=\dot{l}_{3} \cdot \sin \varphi_{10}+l_{3} \cdot \cos \varphi_{10} \cdot \dot{\varphi}_{10}-a \cdot \cos \varphi_{6} \cdot \dot{\varphi}_{6}
\end{array}\right.
\end{array}\right.
$$

For determining of accelerations must to derivate the systems I-IV, but we take in consideration a method rapid and directly: we know now the velocities and one derivate directly their relations; it obtains the relations from the system 5 [11]. 


$$
\begin{aligned}
& \left\{\left\{\ddot{\varphi}_{3}=\frac{\ddot{l}_{1}-\dot{\varphi}_{3} \cdot g \cdot \cos \left(\varphi_{8}-\varphi_{3}-\beta\right) \cdot\left(\dot{\varphi}_{8}-\dot{\varphi}_{3}\right)}{g \cdot \sin \left(\varphi_{8}-\varphi_{3}-\beta\right)}\right.\right. \\
& \int \ddot{\varphi}_{8}=\frac{\cos \left(\varphi_{8}-\varphi_{3}-\beta\right) \cdot \ddot{l}_{1}-\dot{l}_{1} \cdot \sin \left(\varphi_{8}-\varphi_{3}-\beta\right) \cdot\left(\dot{\varphi}_{8}-\dot{\varphi}_{3}\right)}{l_{1} \cdot \sin \left(\varphi_{8}-\varphi_{3}-\beta\right)}- \\
& \left\{-\frac{\dot{\varphi}_{8} \cdot l_{1} \cdot \cos \left(\varphi_{8}-\varphi_{3}-\beta\right) \cdot\left(\dot{\varphi}_{8}-\dot{\varphi}_{3}\right)+\dot{\varphi}_{8} \cdot \dot{l}_{1} \cdot \sin \left(\varphi_{8}-\varphi_{3}-\beta\right)}{l_{1} \cdot \sin \left(\varphi_{8}-\varphi_{3}-\beta\right)}\right. \\
& \int \ddot{\varphi}_{6}=\frac{e \cdot \cos \left(\varphi_{6}-\varphi_{3}\right) \cdot\left(\dot{\varphi}_{6}-\dot{\varphi}_{3}\right) \cdot \dot{\varphi}_{3}+e \cdot \sin \left(\varphi_{6}-\varphi_{3}\right) \cdot \ddot{\varphi}_{3}-\ddot{l}_{2}}{a \cdot \sin \left(\varphi_{10}-\varphi_{6}\right)}- \\
& -\frac{\dot{\varphi}_{6} \cdot a \cdot \cos \left(\varphi_{10}-\varphi_{6}\right) \cdot\left(\dot{\varphi}_{10}-\dot{\varphi}_{6}\right)}{a \cdot \sin \left(\varphi_{10}-\varphi_{6}\right)} \\
& \left\{\ddot{\varphi}_{10}=\frac{\ddot{l}_{2} \cdot \cos \left(\varphi_{10}-\varphi_{6}\right)-\dot{l}_{2} \cdot \sin \left(\varphi_{10}-\varphi_{6}\right) \cdot\left(\dot{\varphi}_{10}-\dot{\varphi}_{6}\right)}{l_{2} \cdot \sin \left(\varphi_{10}-\varphi_{6}\right)}-\right. \\
& \left\{-\frac{e \cdot \cos \left(\varphi_{6}-\varphi_{3}\right) \cdot\left(\dot{\varphi}_{6}-\dot{\varphi}_{3}\right) \cdot \dot{\varphi}_{3}+e \cdot \sin \left(\varphi_{6}-\varphi_{3}\right) \cdot \ddot{\varphi}_{3}}{l_{2} \cdot \sin \left(\varphi_{10}-\varphi_{6}\right)}-\right. \\
& -\frac{\dot{\varphi}_{10} \cdot \dot{l}_{2} \cdot \sin \left(\varphi_{10}-\varphi_{6}\right)+\dot{\varphi}_{10} \cdot l_{2} \cdot \cos \left(\varphi_{10}-\varphi_{6}\right) \cdot\left(\dot{\varphi}_{10}-\dot{\varphi}_{6}\right)}{l_{2} \cdot \sin \left(\varphi_{10}-\varphi_{6}\right)} \\
& \left\{\ddot{\varphi}_{1}=\frac{2 a \cdot \cos \left(\varphi_{6}-\varphi_{1}\right) \cdot \ddot{\varphi}_{6}-2 a \cdot \sin \left(\varphi_{6}-\varphi_{1}\right) \cdot\left(\dot{\varphi}_{6}-\dot{\varphi}_{1}\right) \cdot \dot{\varphi}_{6}-\dot{\varphi}_{1} \cdot \dot{l}_{3}}{l_{3}}\right. \\
& \ddot{x}_{M}=\ddot{l}_{3} \cdot \cos \varphi_{10}-\dot{l}_{3} \cdot \sin \varphi_{10} \cdot \dot{\varphi}_{10}-\dot{l}_{3} \cdot \sin \varphi_{10} \cdot \dot{\varphi}_{10}-l_{3} \cdot \cos \varphi_{10} \cdot \dot{\varphi}_{10}^{2}- \\
& -l_{3} \cdot \sin \varphi_{10} \cdot \ddot{\varphi}_{10}+a \cdot \cos \varphi_{6} \cdot \dot{\varphi}_{6}^{2}+a \cdot \sin \varphi_{6} \cdot \ddot{\varphi}_{6} \\
& \ddot{y}_{M}=\ddot{l}_{3} \cdot \sin \varphi_{10}+\dot{l}_{3} \cdot \cos \varphi_{10} \cdot \dot{\varphi}_{10}+\dot{l}_{3} \cdot \cos \varphi_{10} \cdot \dot{\varphi}_{10}-l_{3} \cdot \sin \varphi_{10} \cdot \dot{\varphi}_{10}^{2}+ \\
& +l_{3} \cdot \cos \varphi_{10} \cdot \ddot{\varphi}_{10}+a \cdot \sin \varphi_{6} \cdot \dot{\varphi}_{6}^{2}-a \cdot \cos \varphi_{6} \cdot \ddot{\varphi}_{6}
\end{aligned}
$$

One determines now and the last kinematics parameters of the mechanism, for to have a complete cinematic of the main mechanism, which is necessary and in the kinetostatic and dynamic calculations (systems 6-21) [11].

$$
\left\{\begin{array}{l}
\left\{\begin{array}{l}
x_{C}=c \cdot \cos \left(\varphi_{3}-C B D\right) \\
y_{C}=c \cdot \sin \left(\varphi_{3}-C B D\right)
\end{array}\right) \begin{array}{l}
\dot{x}_{C}=-c \cdot \sin \left(\varphi_{3}-C B D\right) \cdot \dot{\varphi}_{3} \\
\dot{y}_{C}=c \cdot \cos \left(\varphi_{3}-C B D\right) \cdot \dot{\varphi}_{3}
\end{array} \\
\left\{\begin{array}{l}
\ddot{x}_{C}=-c \cdot \cos \left(\varphi_{3}-C B D\right) \cdot \dot{\varphi}_{3}^{2}-c \cdot \sin \left(\varphi_{3}-C B D\right) \cdot \ddot{\varphi}_{3} \\
\ddot{y}_{C}=-c \cdot \sin \left(\varphi_{3}-C B D\right) \cdot \dot{\varphi}_{3}^{2}+c \cdot \cos \left(\varphi_{3}-C B D\right) \cdot \ddot{\varphi}_{3}
\end{array}\right.
\end{array}\right.
$$




$$
\begin{aligned}
& \left\{\begin{array}{l}
x_{G_{3}}=s_{3} \cdot \cos \left(\varphi_{3}-C B D+\alpha\right) \\
y_{G_{3}}=s_{3} \cdot \sin \left(\varphi_{3}-C B D+\alpha\right)
\end{array}\right)\left(\begin{array}{l}
\dot{x}_{G_{3}}=-s_{3} \cdot \sin \left(\varphi_{3}-C B D+\alpha\right) \cdot \dot{\varphi}_{G_{3}}=s_{3} \cdot \cos \left(\varphi_{3}-C B D+\alpha\right) \cdot \dot{\varphi}_{3} \\
\dot{y}_{G_{3}}
\end{array}\right.
\end{aligned}
$$

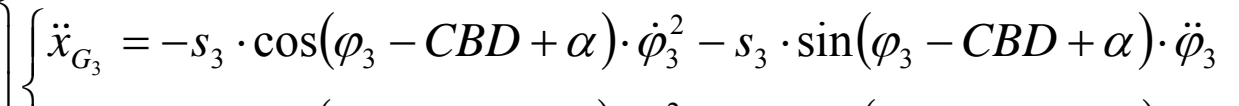

$$
\begin{aligned}
& \left\{\ddot{y}_{G_{3}}=-s_{3} \cdot \sin \left(\varphi_{3}-C B D+\alpha\right) \cdot \dot{\varphi}_{3}^{2}+s_{3} \cdot \cos \left(\varphi_{3}-C B D+\alpha\right) \cdot \ddot{\varphi}_{3}\right. \\
& \left\{\begin{array} { l } 
{ x _ { D } = e \cdot \operatorname { c o s } \varphi _ { 3 } } \\
{ y _ { D } = e \cdot \operatorname { s i n } \varphi _ { 3 } }
\end{array} \left\{\begin{array} { l } 
{ \dot { x } _ { D } = - e \cdot \operatorname { s i n } \varphi _ { 3 } \cdot \dot { \varphi } _ { 3 } } \\
{ \dot { y } _ { D } = e \cdot \operatorname { c o s } \varphi _ { 3 } \cdot \dot { \varphi } _ { 3 } }
\end{array} \left\{\begin{array}{l}
\ddot{x}_{D}=-e \cdot \cos \varphi_{3} \cdot \dot{\varphi}_{3}^{2}-e \cdot \sin \varphi_{3} \cdot \ddot{\varphi}_{3} \\
\ddot{y}_{D}=-e \cdot \sin \varphi_{3} \cdot \dot{\varphi}_{3}^{2}+e \cdot \cos \varphi_{3} \cdot \ddot{\varphi}_{3}
\end{array}\right.\right.\right. \\
& \left\{\left\{\begin{array} { l } 
{ x _ { G _ { 4 } } = x _ { C } + s _ { 4 } \cdot \operatorname { c o s } \varphi _ { 4 } } \\
{ y _ { G _ { 4 } } = y _ { C } + s _ { 4 } \cdot \operatorname { s i n } \varphi _ { 4 } }
\end{array} \left\{\begin{array} { l } 
{ \dot { x } _ { G _ { 4 } } = \dot { x } _ { C } } \\
{ \dot { y } _ { G _ { 4 } } = \dot { y } _ { C } }
\end{array} \left\{\begin{array}{l}
\ddot{x}_{G_{4}}=\ddot{x}_{C} \\
\ddot{y}_{G_{4}}=\ddot{y}_{C}
\end{array}\right.\right.\right.\right.
\end{aligned}
$$

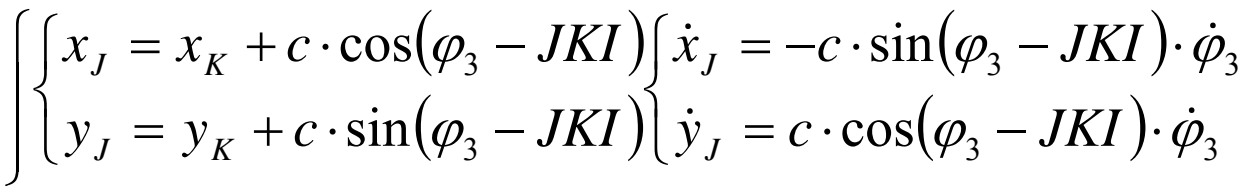

$$
\begin{aligned}
& \left\{\begin{array}{l}
\ddot{x}_{J}=-c \cdot \cos \left(\varphi_{3}-J K I\right) \cdot \dot{\varphi}_{3}^{2}-c \cdot \sin \left(\varphi_{3}-J K I\right) \cdot \ddot{\varphi}_{3} \\
\ddot{y}_{J}=-c \cdot \sin \left(\varphi_{3}-J K I\right) \cdot \dot{\varphi}_{3}^{2}+c \cdot \cos \left(\varphi_{3}-J K I\right) \cdot \ddot{\varphi}_{3}
\end{array}\right. \\
& \left\{\begin{array} { l } 
{ x _ { I } = x _ { K } + e \cdot \operatorname { c o s } \varphi _ { 3 } } \\
{ y _ { I } = y _ { K } + e \cdot \operatorname { s i n } \varphi _ { 3 } }
\end{array} \left\{\begin{array}{l}
\dot{x}_{I}=-e \cdot \sin \varphi_{3} \cdot \dot{\varphi}_{3} \\
\dot{y}_{I}=e \cdot \cos \varphi_{3} \cdot \dot{\varphi}_{3}
\end{array}\right.\right. \\
& \left\{\begin{array}{l}
\ddot{x}_{I}=-e \cdot \cos \varphi_{3} \cdot \dot{\varphi}_{3}^{2}-e \cdot \sin \varphi_{3} \cdot \ddot{\varphi}_{3} \\
\ddot{y}_{I}=-e \cdot \sin \varphi_{3} \cdot \dot{\varphi}_{3}^{2}+e \cdot \cos \varphi_{3} \cdot \ddot{\varphi}_{3}
\end{array}\right.
\end{aligned}
$$

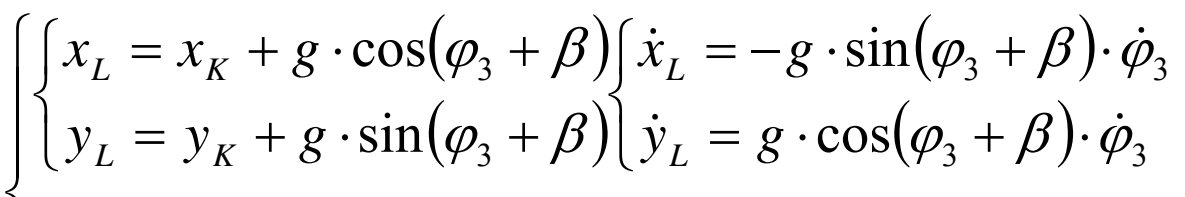

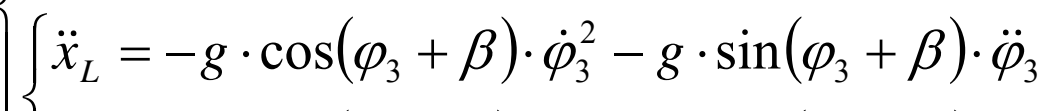

$$
\begin{aligned}
& \left\{\ddot{y}_{L}=-g \cdot \sin \left(\varphi_{3}+\beta\right) \cdot \dot{\varphi}_{3}^{2}+g \cdot \cos \left(\varphi_{3}+\beta\right) \cdot \ddot{\varphi}_{3}\right.
\end{aligned}
$$

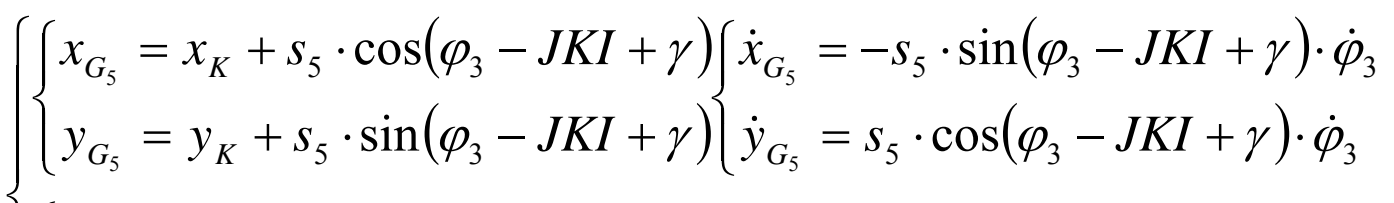

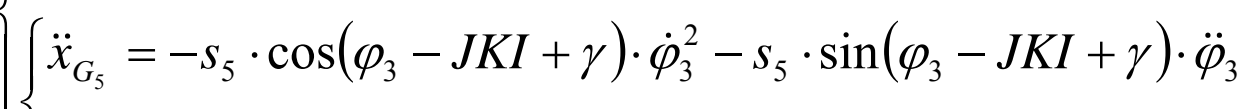

$$
\begin{aligned}
& \ddot{y}_{G_{5}}=-s_{5} \cdot \sin \left(\varphi_{3}-J K I+\gamma\right) \cdot \dot{\varphi}_{3}^{2}+s_{5} \cdot \cos \left(\varphi_{3}-J K I+\gamma\right) \cdot \ddot{\varphi}_{3}
\end{aligned}
$$




$$
\begin{aligned}
& \left\{\begin{array} { l } 
{ x _ { G _ { 8 } } = x _ { F } + \frac { 1 } { 2 } l _ { 1 } \cdot \operatorname { c o s } \varphi _ { 8 } } \\
{ y _ { G _ { 8 } } = y _ { F } + \frac { 1 } { 2 } l _ { 1 } \cdot \operatorname { s i n } \varphi _ { 8 } }
\end{array} \left\{\begin{array}{l}
\dot{x}_{G_{8}}=\frac{1}{2} l_{1} \cdot \cos \varphi_{8}-\frac{1}{2} l_{1} \cdot \sin \varphi_{8} \cdot \dot{\varphi}_{8} \\
\dot{y}_{G_{8}}=\frac{1}{2} \dot{l}_{1} \cdot \sin \varphi_{8}+\frac{1}{2} l_{1} \cdot \cos \varphi_{8} \cdot \dot{\varphi}_{8}
\end{array}\right.\right. \\
& \int \ddot{x}_{G_{8}}=\frac{1}{2} \ddot{l}_{1} \cdot \cos \varphi_{8}-\frac{1}{2} \dot{l}_{1} \cdot \sin \varphi_{8} \cdot \dot{\varphi}_{8}-\frac{1}{2} \dot{l}_{1} \cdot \sin \varphi_{8} \cdot \dot{\varphi}_{8}- \\
& -\frac{1}{2} l_{1} \cdot \cos \varphi_{8} \cdot \dot{\varphi}_{8}^{2}-\frac{1}{2} l_{1} \cdot \sin \varphi_{8} \cdot \ddot{\varphi}_{8} \\
& \ddot{y}_{G_{8}}=\frac{1}{2} \ddot{l}_{1} \cdot \sin \varphi_{8}+\frac{1}{2} \dot{l}_{1} \cdot \cos \varphi_{8} \cdot \dot{\varphi}_{8}+\frac{1}{2} \dot{l}_{1} \cdot \cos \varphi_{8} \cdot \dot{\varphi}_{8}- \\
& -\frac{1}{2} l_{1} \cdot \sin \varphi_{8} \cdot \dot{\varphi}_{8}^{2}+\frac{1}{2} l_{1} \cdot \cos \varphi_{8} \cdot \ddot{\varphi}_{8}
\end{aligned}
$$

$\ddot{y}_{E}=\ddot{y}_{D}-\ddot{l}_{3} \cdot \sin \varphi_{1}-\dot{l}_{3} \cdot \cos \varphi_{1} \cdot \dot{\varphi}_{1}-\dot{l}_{3} \cdot \cos \varphi_{1} \cdot \dot{\varphi}_{1}+$

$+l_{3} \cdot \sin \varphi_{1} \cdot \dot{\varphi}_{1}^{2}-l_{3} \cdot \cos \varphi_{1} \cdot \ddot{\varphi}_{1}$ 


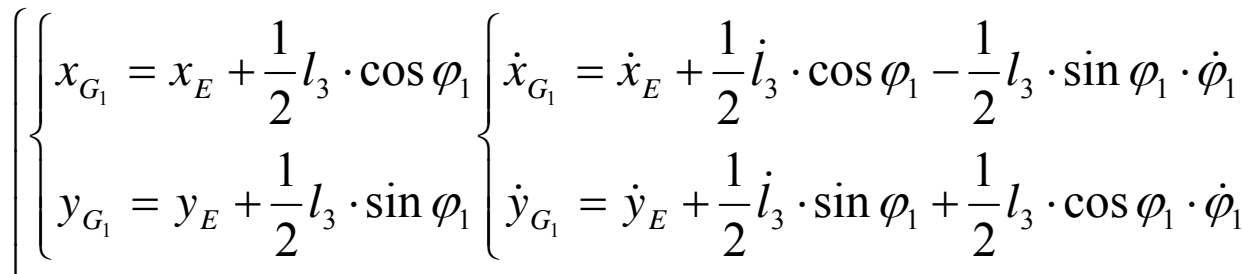

$$
\begin{aligned}
& \int \ddot{x}_{G_{1}}=\ddot{x}_{E}+\frac{1}{2} \ddot{l}_{3} \cdot \cos \varphi_{1}-\frac{1}{2} \dot{l}_{3} \cdot \sin \varphi_{1} \cdot \dot{\varphi}_{1}-\frac{1}{2} \dot{l}_{3} \cdot \sin \varphi_{1} \cdot \dot{\varphi}_{1}- \\
& -\frac{1}{2} l_{3} \cdot \cos \varphi_{1} \cdot \dot{\varphi}_{1}^{2}-\frac{1}{2} l_{3} \cdot \sin \varphi_{1} \cdot \ddot{\varphi}_{1} \\
& \ddot{y}_{G_{1}}=\ddot{y}_{E}+\frac{1}{2} \ddot{l}_{3} \cdot \sin \varphi_{1}+\frac{1}{2} \dot{l}_{3} \cdot \cos \varphi_{1} \cdot \dot{\varphi}_{1}+\frac{1}{2} \dot{l}_{3} \cdot \cos \varphi_{1} \cdot \dot{\varphi}_{1}- \\
& -\frac{1}{2} l_{3} \cdot \sin \varphi_{1} \cdot \dot{\varphi}_{1}^{2}+\frac{1}{2} l_{3} \cdot \cos \varphi_{1} \cdot \ddot{\varphi}_{1} \\
& \left\{\begin{array} { l } 
{ x _ { G } = x _ { I } - 2 a \cdot \operatorname { c o s } \varphi _ { 6 } } \\
{ y _ { G } = y _ { I } - 2 a \cdot \operatorname { s i n } \varphi _ { 6 } }
\end{array} \left\{\begin{array}{l}
\dot{x}_{G}=\dot{x}_{I}+2 a \cdot \sin \varphi_{6} \cdot \dot{\varphi}_{6} \\
\dot{y}_{G}=\dot{y}_{I}-2 a \cdot \cos \varphi_{6} \cdot \dot{\varphi}_{6}
\end{array}\right.\right.
\end{aligned}
$$

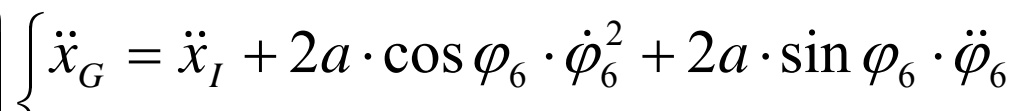

$$
\begin{aligned}
& \ddot{y}_{G}=\ddot{y}_{I}+2 a \cdot \sin \varphi_{6} \cdot \dot{\varphi}_{6}^{2}-2 a \cdot \cos \varphi_{6} \cdot \ddot{\varphi}_{6} \\
& \left\{\begin{array} { l } 
{ x _ { H } \equiv x _ { G _ { 6 } } = x _ { I } - a \cdot \operatorname { c o s } \varphi _ { 6 } } \\
{ y _ { H } \equiv y _ { G _ { 6 } } = y _ { I } - a \cdot \operatorname { s i n } \varphi _ { 6 } }
\end{array} \left\{\begin{array}{l}
\dot{x}_{H}=\dot{x}_{I}+a \cdot \sin \varphi_{6} \cdot \dot{\varphi}_{6} \\
\dot{y}_{H}=\dot{y}_{I}-a \cdot \cos \varphi_{6} \cdot \dot{\varphi}_{6}
\end{array}\right.\right. \\
& \left\{\begin{array}{l}
\ddot{x}_{H}=\ddot{x}_{I}+a \cdot \cos \varphi_{6} \cdot \dot{\varphi}_{6}^{2}+a \cdot \sin \varphi_{6} \cdot \ddot{\varphi}_{6} \\
\ddot{y}_{H}=\ddot{y}_{I}+a \cdot \sin \varphi_{6} \cdot \dot{\varphi}_{6}^{2}-a \cdot \cos \varphi_{6} \cdot \ddot{\varphi}_{6}
\end{array}\right. \\
& \left\{\begin{array} { l } 
{ x _ { G _ { 7 } } = x _ { G } + s _ { 7 } \cdot \operatorname { c o s } \varphi } \\
{ y _ { G _ { 7 } } = y _ { G } + s _ { 7 } \cdot \operatorname { s i n } \varphi }
\end{array} \left\{\begin{array} { l } 
{ \dot { x } _ { G _ { 7 } } = \dot { x } _ { G } } \\
{ \dot { y } _ { G _ { 7 } } = \dot { y } _ { G } }
\end{array} \left\{\begin{array}{l}
\ddot{x}_{G_{7}}=\ddot{x}_{G} \\
\ddot{y}_{G_{7}}=\ddot{y}_{G}
\end{array}\right.\right.\right. \\
& \left\{\begin{array} { l } 
{ x _ { M } = x _ { G } + f \cdot \operatorname { c o s } ( \varphi + \theta ) } \\
{ y _ { M } = y _ { G } + f \cdot \operatorname { s i n } ( \varphi + \theta ) }
\end{array} \left\{\begin{array} { l } 
{ \dot { x } _ { M } = \dot { x } _ { G } } \\
{ \dot { y } _ { M } = \dot { y } _ { G } }
\end{array} \left\{\begin{array}{l}
\ddot{x}_{M}=\ddot{x}_{G} \\
\ddot{y}_{M}=\ddot{y}_{G}
\end{array}\right.\right.\right.
\end{aligned}
$$

\section{APPLICATIONS}

Presented system can be useful in all forging oversized, and in particular to the forging manipulators (independent or on the rail) [1-12]. Railbound forging manipulators are used when is require an accuracy of positioning very high and a greater stability. 
INDEPENDENT JOURNAL OF MANAGEMENT \& PRODUCTION (IJM\&P)

http://www.ijmp.jor.br

v. 6 , n. 3, July - September 2015

ISSN: 2236-269X

DOI: $10.14807 /$ ijmp.v6i3.235

\section{CONCLUSIONS}

Heavy payload forging manipulators are mainly characterized by large load output and large capacitive load input. The relationship between outputs and inputs, which will greatly influence the control and the reliability, is the key issue in type design for heavy payload forging manipulators. Forging manipulators have become more prevalent in the industry today. They are used to manipulate objects to be forged.

The most common forging manipulators are moving on a railway to have a greater precision and stability. They have been called the railbound forging manipulators. In this paper we analyse the general kinematics of the main mechanism from such manipulator.

Kinematic scheme shows a typical forging manipulator, with the basic motions in operation process: walking, motion of the tong and buffering. The lifting mechanism consists of several parts including linkages, hydraulic drives and motion pairs. Hydraulic drives are with the lifting hydraulic cylinder, the buffer hydraulic cylinder and the leaning hydraulic cylinder, which are individually denoted by c1, c2 and c3. In lifting process, the cylinder $\mathrm{c} 1$ controls the vertical movement of work piece through inputting lifting signal. At the same time, the cylinders $c 2$ and $c 3$ are perfectly closed. While c1 and c3 are closed cylinders, cylinder c2 performs horizontal movement. While, the cylinders $\mathrm{c} 1$ and $\mathrm{c} 2$ are closed the cylinder $\mathrm{c} 3$ realizes leaning movement by inputting leaning signal in leaning condition.

In direct kinematics one knows 11,12 and must be determined: intermediary 13 , $\mathrm{FI} 1, \mathrm{FI} 3, \mathrm{FI} 6, \mathrm{Fl}$, FI10 and finally $\mathrm{xM}, \mathrm{yM}$. In inverse kinematics one knows $\mathrm{xM}, \mathrm{yM}$ (imposed) and must be determined FI1, FI3, Fl6, Fl8, Fl10, I1, I2, I3 so that the FI angle keeps its constant value ( $\mathrm{FI}=\mathrm{PI}-\mathrm{TETA})$ to maintain permanently the segment $\mathrm{GM}$ horizontally.

In this work we are solving positions (in inverse kinematics) with systems V, VI. When we know all these parameters (angles and lengths) one may determine all kinematics parameters. The concept of modelling method based on the outputs tasks is defined and investigated. The principle of type design from the viewpoints of the relationship between output characteristics and actuator inputs is discussed. An idea of establishing the incidence relationship between output characteristics and actuator 
INDEPENDENT JOURNAL OF MANAGEMENT \& PRODUCTION (IJM\&P)

http://www.ijmp.jor.br

v. 6, n. 3, July - September 2015

ISSN: 2236-269X

DOI: 10.14807/ijmp.v6i3.235

inputs is proposed. These novel forging manipulators which satisfy certain functional requirements provide an effective help for the design of forging manipulators.

Presented system can be useful in all forging oversized, and in particular to the forging manipulators (independent or on the rail). Railbound forging manipulators are used when is require an accuracy of positioning very high and a greater stability.

\section{REFERENCES}

DANGO \& DIENENTHAL (2015) DANGO \& DIENENTHAL Filtertechnik GmbH. Available: http://www.dds-filter.com/. Access: 20th March, 2015.

GAO, F.; GUO, W. Z. (2009) Coordinated kinematic modeling for motion planning of heavy-duty manipulators in an integrated open-die forging center, Journal of Engineering Manufacture, v. 223, n. 10, p. 1299-1313.

GAO, F.; GUO, W. Z.; SONG, Q. Y.; DU, F. S. (2010) Current Development of Heavy-duty Manufacturing Equipment, Journal of Mechanical Engineering, v. 46, n. 19, 2010, p. 92-107.

GE, H.; GAO, F. (2012) Type Design for Heavy-payload Forging Manipulators, Chinese Journal of Mechanical Engineering, v. 25, n. 2, p. 197-205.

LI, G.; LIU, D. S. (2010) Dynamic Behavior of the Forging Manipulator under Large Amplitude Compliance Motion, Journal of Mechanical Engineering, v. 46, n. 11, p. 21-28.

PETRESCU, F. I.; PETRESCU, R. V. (2013) Cinematics of the 3R Dyad, in journal Engevista, v. 15, n. 2, p. 118-124, August, ISSN 1415-7314. Available from:

http://www.uff.br/engevista/seer/index.php/engevista/article/view/376.

PETRESCU, F. I.; PETRESCU, R. V. (2012) Kinematics of the Planar Quadrilateral Mechanism, in journal Engevista, v. 14, n. 3, p. 345-348, December, ISSN 14157314. Available from:

http://www.uff.br/engevista/seer/index.php/engevista/article/view/377.

PETRESCU, F. I.; PETRESCU, R. V. (2012) Mecatronica - Sisteme Seriale si Paralele, Create Space publisher, USA, March, ISBN 978-1-4750-6613-5, 128 pages, Romanian edition.

PETRESCU, F. I.; PETRESCU, R. V (2011) Mechanical Systems, Serial and Parallel - Course (in romanian), LULU Publisher, London, UK, February, 124 pages, ISBN 978-1-4466-0039-9, Romanian edition.

PETRESCU, F. I.; GRECU, B.; COMANESCU, A.; PETRESCU, R. V. (2009) Some Mechanical Design Elements. In the 3rd International Conference on Computational Mechanics and Virtual Engineering, COMEC 2009, Braşov, October, ISBN 978-973-598-572-1, Edit. UTB, p. 520-525.

PETRESCU, F. I. (2014) Sisteme mecatronice seriale, paralele și mixte. Create Space publisher, USA, February, ISBN 978-1-4959-2381-4, 224 pages, Romanian edition. 
ISSN: 2236-269X

DOI: 10.14807/ijmp.v6i3.235

ZHAO, K.; WANG, H.; CHEN, G. L.; LIN, Z. Q.; HE, Y. B. (2010) Compliance Process Analysis for Forging Manipulator, Journal of Mechanical Engineering, v. 46 , n. 4 , p. 27-34. 